08

\title{
Дефектная структура эпитаксиальных слоев III-нитридов на основе анализа формы рентгенодифракционных пиков
}

\author{
(C) P.T. КютT \\ Физико-технический институт им. А.Ф. Иофрфе РАН, \\ 194021 Санкт-Петербург, Россия \\ e-mail: r.kyutt@mail.ioffe.ru
}

(Поступило в Редакцию 28 сентября 2016 г.)

\begin{abstract}
Проведены экспериментальные исследования формы рентгенодифракционных эпитаксиальных слоев с большой плотностью дислокаций. Измерения проводились на рентгеновском дифрактометре в двух- и трехкристальном вариантах с использованием как $\mathrm{CuK} \alpha$-, так и $\mathrm{MoK} \alpha$-излучения. Объектами исследования были эпитаксиальные слои $\mathrm{GaN}, \mathrm{AlN}, \mathrm{AlGaN}, \mathrm{ZnO}$ и др., выращенные разными методами на подложках сапфира, кремния и карбида кремния и имеющие разную степень структурного совершенства. Толщина слоев варьировала для разных систем от 0.5 до $30 \mu \mathrm{m}$. Показано, что в центральной части пиков они хорошо аппроксимируются функцией Войта с различной долей лоренцовской составляющей, а на крыльях интенсивность спадает быстрее и может быть выражена степенной функцией, при этом показатель не одинаков для разных структур. Имеет место явная зависимость от упорядоченности дислокаций. Для большинства структур с регулярной системой и регулярными прорастающими дислокациями падение интенсивности близко к теоретически предсказанному закону $\Delta \theta^{-3}$, для пленок с хаотическим распределением оно значительно быстрее. Рассмотрена также связь формы пиков от порядка отражения, геометрии дифракции, толщины эпитаксиальных слоев.
\end{abstract}

DOI: 10.21883/JTF.2017.04.44319.2044

\section{Введение}

Параметры дефектной структуры сильнонарушенных эпитаксиальных слоев обычно определяются на основе мозаично-блочной модели путем анализа полуширины дифракционных пиков (графики Вильямсона-Холла). В последние годы возобновился интерес также к анализу формы рентгеновских дифракционных пиков, забытый со времен работ Гордона-Авербаха [1]. Известно, что дислокации представляют собой дефекты второго класса (по классификации Кривоглаза [2]), поскольку слабое спадание полей смещений вокруг них $(\propto 1 / r)$ приводит к тому, что дальние дислокации оказывают большее влияние на дифракционную кривую, чем близлежащие, что значительно затрудняет теоретические расчеты. Кривоглаз в своей основополагающей монографии [2], рассматривая дифракцию от кристаллов с большой плотностью дислокаций и при этом использовав ряд значительных приближений, получил для дифракционной кривой форму гауссиана, но на крыльях вдали от центра интенсивность спадала по степенному закону $1 / \delta \theta^{-3}$. Последующие теоретические работы, где расчеты были в основном построены на методе Монте-Карло, показали несколько иные результаты.

Здесь следует отметить, что в настоящее время рассматриваются обычно два участка дифракционных кривых - центральная пиковая часть и интенсивность на крыльях. При этом сама кривая аппроксимируется в основном функцией Войта (см., например, [3]). Особые различия в разных работах наблюдались на спаде кривых. Расчеты, проведенные Каганером с соавторами [4-6], показали в общем-то классическое распреде- ление интенсивности - гауссовское распределение в центре и степенной закон спадания на крыльях. Для двукристальных пиков при дифракции от эпитаксиальных слоев $\mathrm{GaN}$, снятых с широко открытым окном детектора, это была зависимость $(\Delta \theta)^{-3}$, для кривых $\theta$-сканирования $-(\Delta \theta)^{-4}[4]$. Эти закономерности были подтверждены экспериментальными измерениями, сделанными, правда, для ряда отражений в наклонной геометрии, где основное влияние на дифракцию оказывают прорастающие краевые дислокации. Влияние винтовых дислокаций, решающее в симметричной брэггговской геометрии, не рассматривалось. Аналогичные закономерности наблюдались для системы GaAs/Si [6].

С другой стороны, в работах Холи и его соавторов получены несколько иные результаты. В $[7,8]$ для слоев $\mathrm{AlGaN}$ асимптотическое поведение на крыльях дифракционных пиков симметричного отражения соответствовало степенной зависимости $q^{-n}$, где $n$ для симметричных брэгговских отражений варьировало между 2 и 3 в зависимости от плотности дислокаций (отличие от чистой третьей степени авторы объясняют учетом интегрирования в $q_{y}$-направлении). Из этого авторы делают вывод о превалирующем влиянии прорастающих винтовых дислокаций. В отличие от этого падение интенсивности для дифракции скользящего падения, где рассеяние определяется краевыми прорастающими дислокациями, лежит между $q^{-1}$ и $q_{-2}$ [8]. Последний результат сильно отличается от того, который наблюдался в работе [4].

В общем, в большинстве работ, имеющих дело с формой уширенных (некогерентных) дифракционных пиков, признается, что в центре интенсивность ведет себя как 
гауссиан с некоторой долей лоренциана, а на крыльях спадает по степенному закону.

Но это относится к эпитаксиальным слоям с выраженной дислокационной структурой. Как показано в работе [9], где была измерена дифракция от системы $\mathrm{MnF}_{2}-\mathrm{CaF}_{2}$ на $\mathrm{Si}(001)$, для верхнего слоя, который имел блочную структуру из разных фаз $\left(\mathrm{MnF}_{2}\right.$ имеет ромбическую сингонию), дифракционные пики от симметричных брэгговских отражений никаких степенных крыльев не имели и хорошо аппроксимировались функцией Войта во всем интервале. С другой стороны, однофазный нижний слой $\mathrm{CaF}_{2}$ характеризовался чисто дислокационной структурой и для него имели место закономерности, описанные выше. Кроме того, в настоящей работе было найдено, что для эпитаксиального слоя $\mathrm{GaN}$ падение интенсивности на крыльях зависит от регулярности в распределении дислокаций. Для прямолинейных прорастающих дислокаций интенсивность спадает как $q^{-3}$, а для слоев с хаотическим распределением дислокаций заметно быстрее.

Однако разница во влиянии винтовых и краевых прорастающих дислокаций (и есть ли она) до сих пор остается неясной, что требует дальнейших исследований в этой области.

\section{1. Методика}

В настоящей работе проведено детальное исследование формы дифракционных пиков от сильнодислокационных эпитаксиальных слоев и рассмотрены возможные факторы, которые могут влиять на форму дифракционных линий: тип дислокационной структры, плотность и распределение дислокаций в слоях (и, следовательно, степень уширения пиков), толщина слоя, порядок рентгеновского отражения, геометрия дифракции и материал подложки. Путем набора статистических данных при измерении серий образцов и их анализа проводился поиск закономерностей связи между этими факторами и параметрами формы пиков.

Объектами исследования были эпитаксиальные слои $\mathrm{GaN}, \mathrm{AlN}, \mathrm{AlGaN}, \mathrm{ZnO}$ и др., выращенные разными методами на подложках сапфира, кремния и карбида кремния. Толщина слоев варьировала для разных систем от 0.5 до $20 \mu \mathrm{m}$. Измерения были выполнены на рентгеновском дифрактометре как в двукристальном, так и трехкристальном вариантах. С использованием как $\mathrm{CuK} \alpha$-, так и $\mathrm{MoK} \alpha$-излучений были измерены дифракционные кривые в симметричной брэгговской геометрии и симметричной лауэ-геометрии в двух направлениях сканирования - параллельно дифракционному вектору $(\theta-2 \theta$-сканирование) и перпендикулярно ему ( $\theta$-сканирование).

Дифракционные пики аппроксимировались функцией Войта. Падение интенсивности на хвостах определялось из построения кривых в двойном логарифмическом масштабе. Инструментальное уширение имеет место в основном на пиках $\theta-2 \theta$-моды, и оно учитывалось. Учитывалось также возможное влияние кривизны структур на $\theta$-кривые.

\section{2. Результаты и их обсуждение}

\section{1. Симметричная брэгговская геометрия}

На рис. 1-3 представлены двукристальные дифракционные кривые для трех эпитаксиальных слоев как в полулогарифмическом, так и в двойном логарифмическом масштабах. Из кривых видно, что центральная часть не характеризуется чистым гауссианом, а аппроксимируется функцией Войта, содержащей лоренцовскую составляющую. Доля последней в большинстве случаев заметно меньше, хотя отношение вкладов лоренциана и гауссиана неодинаково для разных образцов. Причина этих различий в настоящее время не ясна, по крайней мере каких-либо закономерностей в этой величине не обнаружено.
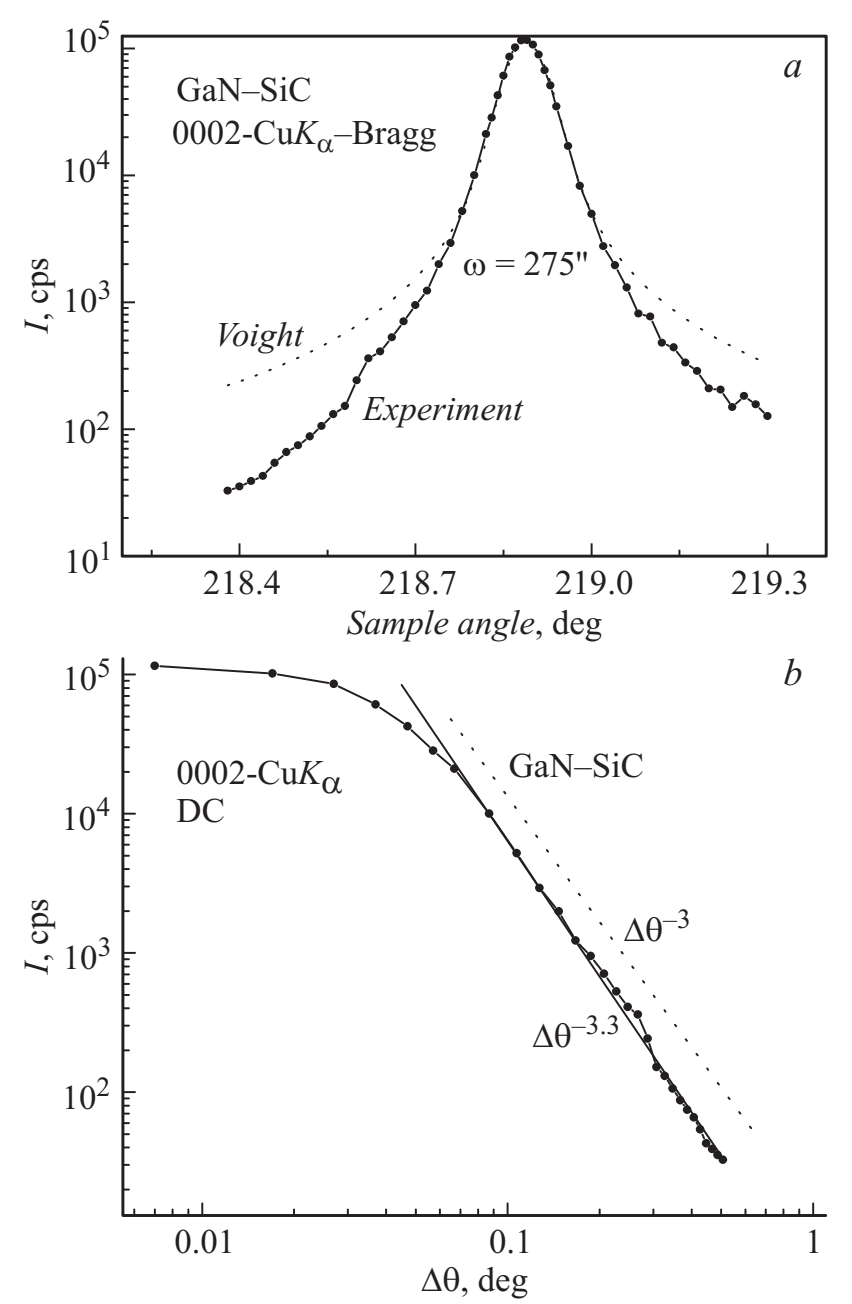

Рис. 1. Двукристальная дифракционная кривая брэгговского отражения 0002 от структуры $\mathrm{GaN}-\mathrm{SiC}$ (толщина пленки $3.5 \mu \mathrm{m})$ в полулогарифмическом $(a)$ и двойном логарифмическом (b) масштабах. 



Рис. 2. Двукристальная дифракционная кривая брэгговского отражения 0002 от структуры $\mathrm{AlN}$-сапфир (толщина пленки $1.2 \mu \mathrm{m})$ в полулогарифмическом $(a)$ и двойном логарифмическом $(b)$ масштабах.

Здесь надо отметить, что уширение, перпендикулярное дифракционному вектору, зависит только от дефектной структуры, для идеальных кристаллических слоев оно определяется только инструментальными факторами.

Для большинства структур падение интенсивности на хвостах кривых отклоняется от пиковой функции (оно более быстрое) и может быть аппроксимировано степенной функцией. И в этой части имеет место значительное расхождение между разными образцами. При этом в выражении $\Delta \theta^{-n}$ показатель $n$ может варьировать от 2 до 5. И здесь наблюдается достаточно четкая закономерность. Для слоев с регулярной системой прорастающих прямолинейных дислокаций поведение интенсивности на хвостах практически равно теоретически предсказанной Кривоглазом и развитой в работах Каганера с соавторами - это $n=3$ для двукристальной кривой и $n=4$ для пиков $\theta$-сканирования. Поскольку, как известно, подавляющий вклад в уширение брэгговских симметричных кривых вносят перпендикулярные поверхности прямолинейные винтовые дислокации, можно сказать, что для них это экспериментально доказанная закономерность.

С другой стороны, для пленок с хаотическим распределением дислокаций по объему интенсивность спадает на крыльях кривых много быстрее. Такое распределение было доказано как снимками поперечных срезов в электронной микроскопии, так и сравнением ширины дифракционных пиков в брэгговской и лауэвской геометрии [10], или сравнением полуширин брэгговских кривых асимметричных отражений, снятых при скользящем падении и скользящем отражении. Пример такой кривой приведен на рис. 3. Очевидно, здесь большую роль играют горизонтальные фрагменты дислокаций.

Что касается кривых $\theta-2 \theta$-сканирования (параллельно дифракционному вектору), то здесь трудно проследить какие-либо закономерности. Кривые $\theta-2 \theta$-сканирования в брэгговской дифракции для большинства дислокационных эпитаксиальных слоев (естественно, не очень тонких, чтобы не сказалось уширение из-за толщи-


Рис. 3. Двукристальная дифракционная кривая брэгговского отражения 0004 от структуры $\mathrm{GaN}$-сапфир (толщина пленки $30 \mu \mathrm{m})$ в полулогарифмическом $(a)$ и двойном логарифмическом $(b)$ масштабах. 

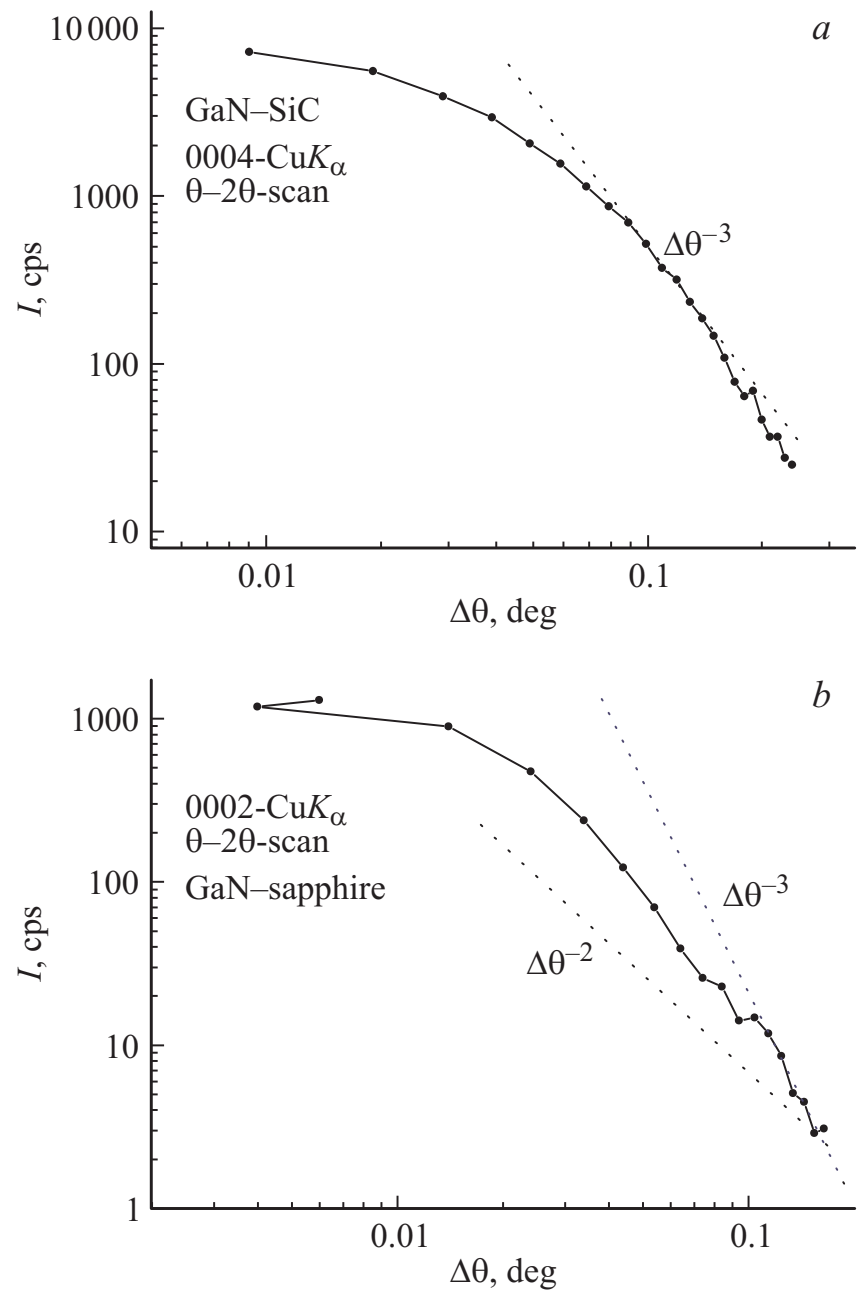

Рис. 4. Распределение интенсивности в симметричной брэгговской дифракции в направлениии $\theta-2 \theta-$ вдоль дифракционного вектора для двух эпитаксиальных пленок $\mathrm{GaN}$.

ны) значительно меньше, чем уширение в поперечном направлении (из-за локальных разориентаций). Угловая ширина $\theta-2 \theta$-пиков несильно различается для пленок с различным уровнем дефектности (плотности дислокаций) (в пределах 60-90 угловых секунд), и уширение, очевидно, вызвано иными, чем в поперечном направлении, причинами. Если исключить влияние толщины слоя, то из общих соображений это уширение связано с вариацией расстояний между плоскостями, параллельными поверхности (компонента микродисторсии $e_{z z}$ ), и поэтому можно ожидать, что и падение интенсивности на крыльях таких кривых может быть иным. И действительно, наши эксперименты показывают, что интенсивность на хвостах в радиальном направлении спадает заметно медленнее, чем в поперечном (пример приведен на рис. 4).

Здесь надо отметить, что для совершенных кристаллов (пластин, слоев) падение интенсивности вдоль дифракционного вектора (симметричная брэгговская геометрия) подчиняется закону $\Delta \theta^{-2}$ как по динамической теории (и в вариации Дарвина, и в вариации Лауэ), так и по кинематической (независимо от толщины пленки). Отклонение от этого закона, естетственно, должно быть связано с дефектами, если речь идет о чистых соединениях, а не о твердых растворах типа $\mathrm{AlGaN}$. В последнем случае оно может быть обусловлено вариацией состава. Для наших же экспериментальных кривых интенсивность может спадать с разным уровнем степенной зависимости. На такую же неопределенность с кривыми $\theta-2 \theta$-моды ссылались и авторы работы [7]. С другой стороны, в [4] расчетами было показано, что поведение на хвостах тоже удовлетворяет закону $\Delta \theta^{-4}$, правда, вдали от центра пика.

При переходе к другому порядку отражения (в нашем случае к 0004) наблюдаемые закономерности сохраняются. Поскольку при этом происходит только увеличение глубины проникновения, отсюда можно сделать вывод о большей или меньшей равномерности в плотности дислокаций и их распределении по глубине слоя.

Насколько степенная зависимость интенсивности на хвостах двукристальных кривых зависит (или не зависит) от общей степени уширения (полуширины брэгговских пиков), видно из рис. 5. На нем сплошными точками приведены значения, относящиеся к регулярному распределению прямолинейных дислокаций (что, как сказано выше, следует из сравнения брэгговских угловых полуширин пиков с лауэвскими, первые значительно шире), а полыми точками - значения для слоев с хаотическим распределением дислокаций (брэгговские и лауэвские полуширины равны между собой). Из этого рисунка следует несколько выводов. Во-первых, для структур с хаотическими дислокациями спадание интенсивности на хвостах происходит значительно быстрее (подтверждение того, о чем сказано выше). Во-вторых, для большинства образцов имеет место предсказанная

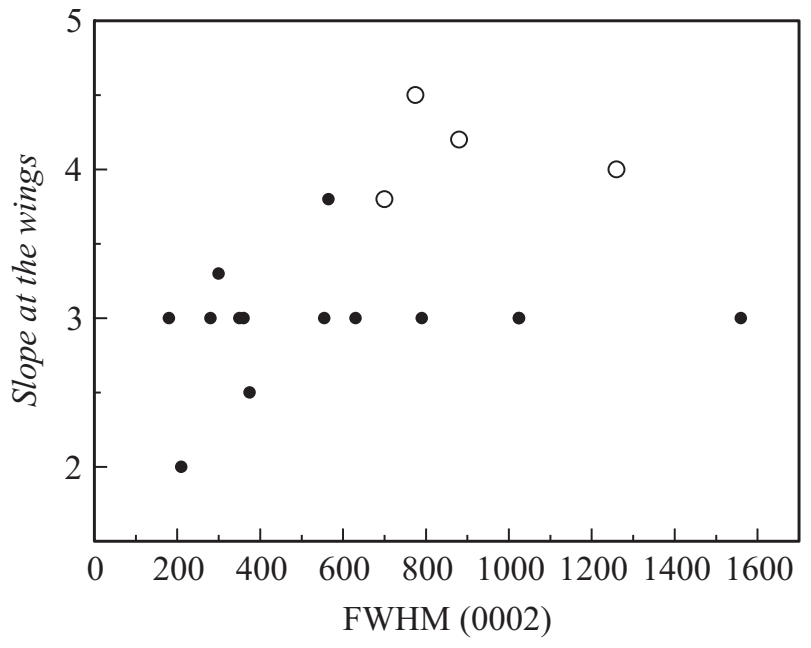

Рис. 5. Показатель степенной зависимости спадания интенсивности на крыльях двукристальных дифракционных кривых брэгговского отражения 0002 для различных образцов с разной степенью уширения, перпендикулярного дифракционнному вектору. 
теорией степенная зависимость $\Delta \theta^{-3}$. Поскольку эта зависимость наблюдается для слоев как с большим, так и с малым уширением, а последнее вызвано только винтовыми прорастающими дислокациям, отсюда следует, что поведение интенсивности на хвостах не зависит от плотности винтовых дислокаций.

Несколько странными оказываются две точки, лежащие ниже уровня -3 , они соответствуют слоям с малой плотностью дислокаций (уширение небольшое). Возможно, в этих структурах плотность дислокаций мала и не оказывает существенного влияния на форму пиков, а падение интенсивности на крыльях вызвано другими дефектами.

Не наблюдается также зависимости закона спадания от состава слоев. Если выделить на рис. 4 точки, относящиеся к $\mathrm{AlN}$ и $\mathrm{AlGaN}$ (не приводится), то для них имеет место та же картина, что и для слоев $\mathrm{GaN}$, т.е. полное отсутствие какой-либо монотонной зависимости. То же самое можно сказать и о влиянии материала подложки: слои, выращенные на сапфире и на $\mathrm{SiC}$, закономерно друг от друга не отличаются.

\section{2. Лауэ-дифракция}

Прорастающие винтовые дислокации не должны влиять на лауэ-пики. С другой стороны, влияние краевых прорастающих дислокаций носит противоположный брэгговскому случаю характер. А именно эти дислокации вносят вклад в уширение дифракционных кривых $\theta-2 \theta$-сканирования из-за дилатаций (изменения межплоскостных расстояний), перпендикулярных поверхности кристаллографических плоскостей, и не вызывают их локальных разворотов, т.е. не влияют на $\theta$-кривые.

На рис. 6 показаны две дифракционные кривые (только в двойном логарифмическом масштабе) от одного из образцов, измеренные в брэговской и лауэ-геометрии, из которых видно, что характер спадания интенсивности на хвостах одинаков в обеих геометриях. Однако данный образец $(\mathrm{AlGaN}-\mathrm{sapph}, x=0.41)$ имел большую плотность хаотически распределенных дислокаций (полуширина двукристальных кривых в обеих геометриях была практически равна, около 1200”).

Таблица 1. Сравнение поведения интенсивности на хвостах дифракционных кривых в брэгговской и лауэ-геометрии

\begin{tabular}{l|c|c|c|c}
\hline \multirow{2}{*}{ Образец } & \multicolumn{2}{|c|}{$10-10$-Laue } & \multicolumn{2}{c}{ 0002-Bragg } \\
\cline { 2 - 5 } & FWHM & Slope & FWHM & Slope \\
\hline AlN-sapph & 135 & -1.5 & 210 & -20 \\
AlGaN-sapph & 1260 & -4.0 & 1300 & -4.0 \\
GaN-SiC & 160 & -2.5 & 300 & -3.3 \\
GaN-Si & 660 & -3.0 & 555 & -4.5 \\
GaN-sapph & 130 & -2.0 & 180 & -3.0 \\
GaN-sapph & 800 & -3.2 & 690 & -4.0 \\
GaN-sapph & 800 & -3.0 & 775 & -4.5
\end{tabular}
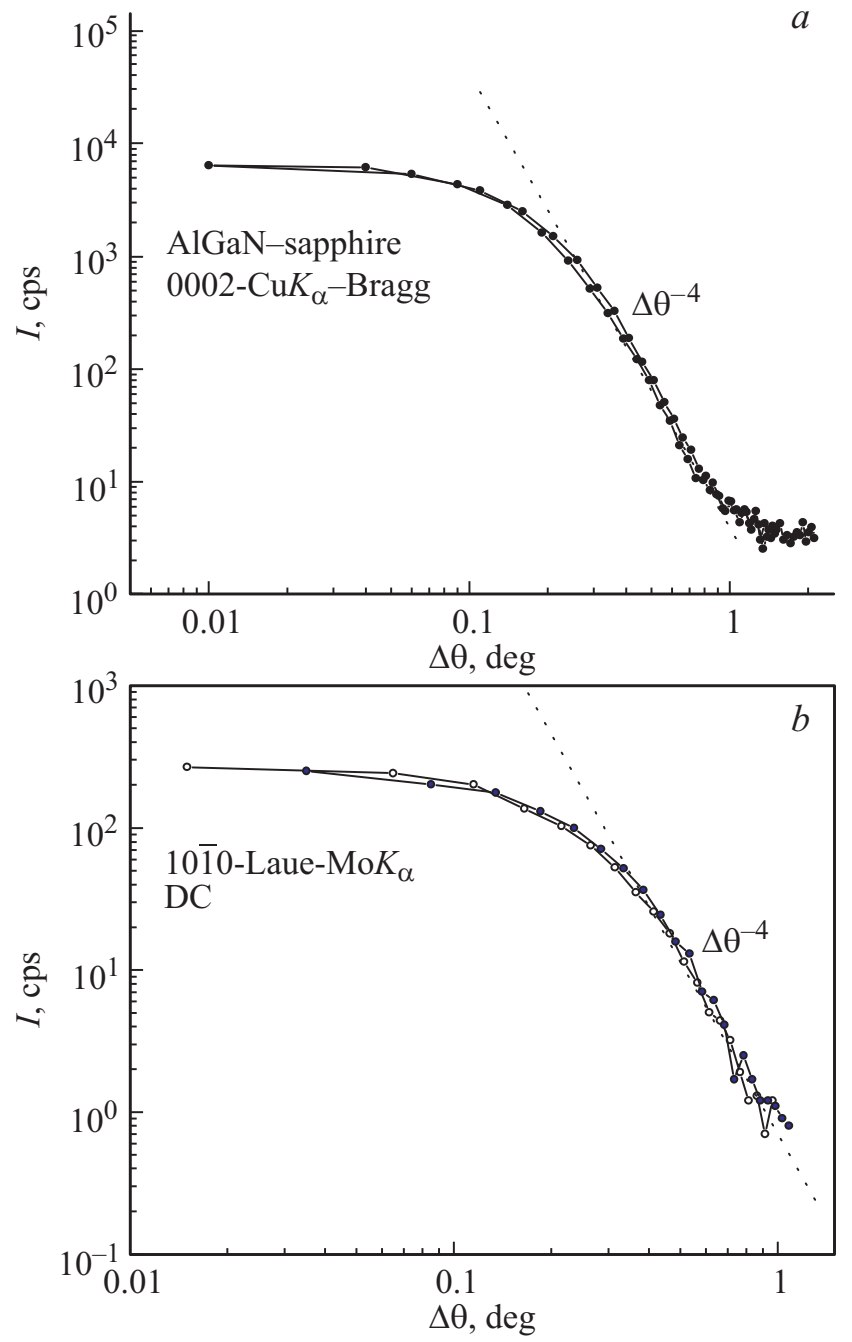

Рис. 6. Спадание интенсивности на хвостах дифракционных кривых в брэгговской (отражение 0002) и лауэвской геометриях (отражение 10-10) для структуры $\mathrm{AlGaN}$-сапфир (толщина слоя $1.5 \mu \mathrm{m})$.

Что же касается структур с системой прямолинейных прорастающих дислокаций, то для них степенная зависимость на лауэ-кривых несколько ниже, чем на брэгговских, хотя она отражает, в общем, те же закономерности, которые имеют место в брэгговской геометрии. Кроме того, в лауэ-дифракции пики $\theta$-сканирования содержат большую долю лоренцовского вклада. В табл. 1 приведены показатели степенной зависимости на хвостах для нескольких образцов в брэгговской и лауэвской геометриях. Объяснение такой разницы может состоять в том, что в полуширину лауэвских двукристальных кривых примерно одинаковый вклад вносят как радиальное, так и поперечное уширения. Здесь надо отметить, что влияние дислокаций на уширение дифракционных пиков в направлении $H(\theta-2 \theta$-сканирование) значительно меньше, чем в поперечном направлении ( $\theta$-сканирование), так что краевые прорастающие дислокации, которые действуют в первом направлении, вносят не 
Таблица 2. Наклон спадания интенсивности на крыльях дифракционнных кривых для эпитаксиальных пленок разной толщины

\begin{tabular}{l|c|c|c}
\hline Образец & Толщина, $\mu \mathrm{m}$ & $\begin{array}{c}\text { FWHM (0002), } \\
\operatorname{arcsec}\end{array}$ & Наклон \\
\hline AlN-sapph & 0.7 & 1560 & -3.0 \\
AlGaN-sapph & 1.65 & 1260 & -4.0 \\
GaN-SiC & 4.5 & 300 & -3.0 \\
GaN-sapph & 12 & 400 & -3.3 \\
GaN-sapph & 18.5 & 800 & -3.0 \\
GaN-sapph & 30 & 775 & -5.0 \\
GaN-sapph & $>50$ & 880 & -4.2
\end{tabular}

такой уж большой вклад, несмотря на их большую плотность. Уширение же в поперечном направлении также существует, хотя его и нельзя увязать с перпендикулярными прорастающими дислокациями как одного, так и другого типов. Его можно охарактеризовать как общее мозаичное уширение.

Что касается возможного уширения пиков за счет ограниченных латеральных размеров областей когерентного рассеяния, то в лауэ-геометрии оно влияет на кривые $\theta-2 \theta$-сканирования. Однако детальные исследования, проведенные ранее $([10])$ с помощью графиков Вильямсона-Холла, показали, что этот вклад мал по сравнению с вкладом краевых прорастающих дислокаций.

\section{3. Зависимость от толщины слоя}

Форма дифракционных кривых, особенно двукристальных и кривых $\theta$-сканирования, никак не зависит от толщины эпитаксиальных слоев исследованных структур. Это хорошо видно из табл. 2, где приведены экспериментальные данные (значения наклонов степенной зависимости) для брэгговских двукристальных кривых для слоев разной толщины.

При хаотическом распределении возрастает вклад горизонтальных фрагментов дислокаций, поэтому более быстрое падение интенсивности на хвостах кривых можно связать именно с ними. То же самое имеет место и для лауэ-дифракции, для которой относительный вклад лоренцовского уширения заметно больше, чем в случае Брэгга.

\section{Заключение}

В настоящей работе собран большой набор экспериментальных данных, касающихся формы дифракционных пиков от сильнарушенных эпитаксиальных слоев. Однако каких-либо четких закономерностей, за некоторым исключением, в зависимости от структурных свойств материалов не просматривается. Прежде всего зададимся вопросом: какой основной тип дефектов влияет на распределение интенсивности? По общему мнению это дислокации, ведь именно они вызывают уширение дифракционных пиков. Классическая дислокационная структура пленок III-нитридов, неоднократно подтвержденная прямыми электронно-микроскопическими наблюдениями, состоит из системы прямолинейных дислокаций, прорастающих сквозь пленку перпендикулярно гетерогранице, винтовых (вектор Бюргерса $b 1=[0001])$, краевых $(b 2$ типа $\langle 11-20\rangle)$ смешанных $(b=\langle 11-23\rangle)$, вклад которых можно разделить на вклады винтовых и краевых. Такая простая дислокационная конструкция не объясняет всех особенностнй дифракционных кривых. С уверенностью из наших данных можно только констатировать свойства винтовых дислокаций, для которых закон спадания интенсивости не зависит ни от плотности дислокаций, ни от толщины эпитаксиального слоя. Если оставаться в жестких рамках только дислокационного вклада, то для объяснения некоторых параметров следует учитывать горизонтальные составляющие дислокаций. Однако для пленок с регулярной системой прорастающих дислокаций горизонтальные фрагменты не просматриваются, а для структур с хаотическим распределением дислокаций по глубине можно рассматривать только некие проекции линий дислокаций на горизонтальную плоскость. Кроме того, поведение интенсивности на хвостах, вообще говоря, обусловлено не направлением линий дислокаций, а полем смещений ближайших дислокаций. Вероятнее всего, быстрое падение интенсивности при хаотическом распределении дислокаций должно быть связано с некой экранировкой полей отдельных семейств дислокаций. Здесь нельзя исключать и влияния других типов дефектов.

\section{Список литературы}

[1] Warren B.E., Averbach B.L. // J. Appl. Phys. 1950. Vol. 21. P. 595.

[2] Кривоглаз М.А.// Теория рассеяния рентгеновских лучей и тепловых нейтронов реальными кристаллами. М.: Наука, 1987. $362 \mathrm{c}$.

[3] Kirste L., Pavlov K.M., Mudie S.T., Punegov V.I., Herres N. // J. Appl. Cryst. 2005. Vol. 38. P. 183.

[4] Kaganer V.M., Brandt O., Trampert A., Ploog K.H. // Phys. Rev. B. 2005. Vol. 72. P. 045448.

[5] Kaganer V.M., Shalimov A., Bak-Misiuk J., Ploog K.H. // Appl. Phys. Lett. 2006. Vol. 89. P. 021922.

[6] Kaganer V.M., Shalimov A., Bak-Misiuk J., Ploog K.H. // J. Phys. Condenc. Matter. 2006. Vol. 18. P. 5047.

[7] Barchuk M., Holy V., Miljevich B., Krause B., Baumbach T., Hertkorn J., Scholz F. // J. Appl. Phys. 2010. Vol. 108. P. 043521.

[8] Lazarev S., Barcyuk M., Bauer S., Forghani K., Holy V., Sholz F., Baumbach T. // J. Appl. Cryst. 2013. Vol. 46. P. $120-127$.

[9] Kyutt R.N., Banshchikov A.G., Kaveev A.K., Sokolov N.S., Ohtake Y., Tabuchi M., Takeda Y., Lomov A.A. // J. Phys. D. Appl. Phys. 2007. Vol. 40. P. 4896.

[10] Ratnikov V.V., Kyutt R.N., Shubina T.V., Pashkova T., Monemar B. // J. Phys. D. Appl. Phys. 2001. Vol. 34. P. 30. 